\title{
Editorial
}

Onkologe 2014 20 :64-65

DOI 10.1007/s00761-013-2543-8

Online publiziert: 22. Januar 2014

c) Springer-Verlag Berlin Heidelberg 2014

\author{
U. Wedding ${ }^{1} \cdot$ K.K. Richter ${ }^{2} \cdot$ B. van Oorschot ${ }^{3} \cdot$ K. Höffken ${ }^{4} \cdot$ P.M. Schlag ${ }^{5}$. \\ M. Bamberg ${ }^{6}$ \\ ${ }^{1}$ Abteilung für Palliativmedizin, Klinik für Innere Medizin II, Universitätsklinikum Jena \\ 2 Dept. of Surgery, Southland Hospital, Invercargill, New Zealand \\ ${ }^{3}$ Palliativstation, Klinik und Poliklinik für Strahlentherapie, Universitätsklinikum Würzburg \\ ${ }^{4}$ Universitätsklinikum Jena \\ ${ }^{5}$ Berlin \\ ${ }^{6}$ Universitätsklinikum Tübingen
}

\section{Neue Rubrik - Palliativmedizin und Supportivtherapie}

Die Herausgeber haben sich entschlossen, die Zeitschrift Der Onkologe um die Rubrik „Palliativmedizin und Supportivtherapie“ zu ergänzen. Dies soll den Lesern die umfassende und ganzheitliche Betreuungsnotwendigkeit onkologischer Patienten besser in den Blick rücken.

Craig E. Earle, Palliativmediziner in Toronto, titulierte sein kürzlich im Journal of Clinical Oncology erschienenes Editorial in Anlehnung an ein afrikanisches Sprichwort, das übersetzt heißt:

\section{It takes a village to raise a child.}

Daraus modifizierte er "It takes a village to help our patients through the journey of their disease"[1]. Der Palliativmediziner ergänzt innerlich gleich „, and their relatives" [1].

Die Aufgaben in der Versorgung onkologischer Patienten sind vielfältig und nicht von einer Disziplin und einer Profession alleine zu tragen. Auch wissen wir aus der Palliativmedizin, dass das betreuende Team die wichtigste Ressource ist, um die mit der Arbeit verbundenen Belastungen gut tragen zu können [2]. Die Vielfalt onkologischer Therapiemöglichkeiten, die aufgrund der demographischen Entwicklung zunehmende Zahl der zu betreuenden Patienten und die exponentielle Zunahme des Wissens führen zu einer zunehmenden Spezialisierung der Onkologen auf Tumorentitäten. Aber auch im Bereich supportiver Möglichkeiten steigt die Zahl relevanter Publikationen und Themenfelder.

\section{1) Wesentliche Elemente der Palliativmedizin sind ganzheitliche und multiprofessionelle Betreuung}

Die moderne Palliativmedizin war zunächst als Betreuung am Lebensende in Strukturen außerhalb der etablierten Versorgung entstanden. Wesentliche Elemente sind die ganzheitliche und multiprofessionelle Betreuung. Zunehmend zeigt sich, dass eine frühe Verankerung palliativer Konzepte begleitend zur onkologischen Betreuung sinnvoll ist.

National [3], europäisch [4] und international [5] ist die Notwendigkeit der Integration der palliativen und supportiven Therapie in die onkologische Betreuung wiederholt betont worden. So will Der Onkologe dazu beitragen, durch die Vermittlung von Wissen, Können und Haltung die Versorgung onkologischer Patienten zu verbessern.

Die geplanten Beiträge zur palliativen Versorgung und supportiven Therapie orientieren sich $\mathrm{z}$. T. an den Schwerpunktthemen; es werden aber auch unabhängig davon Themen als Fortbildungsbeiträge, 
kommentierte Referate und Kongressberichte veröffentlicht werden. Der erste Beitrag soll eine Standortbestimmung zum Thema sein, da die Abgrenzung und Überschneidung von palliativer Versorgung, supportiver Therapie und Hospiz auch im internationalen Schrifttum immer wieder kontrovers ist. Es folgen Beiträge über Strukturen palliativer Versorgung, Modelle zur Integration von onkologischer und palliativer Versorgung und Kriterien einer guten palliativen Betreuung („standard palliative care“).

Wir wünschen Ihnen eine interessante Lektüre

Rubrikherausgeber

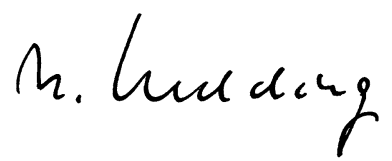

U. Wedding

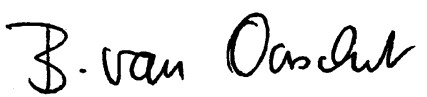

B. van Oorschot

\section{Korrespondenzadresse}

PD Dr. U. Wedding

Abteilung für Palliativmedizin, Klinik für Innere Medizin II, Universitätsklinikum Jena

Erlanger Allee 101, 07747 Jena

ulrich.wedding@med.uni-jena.de

\section{Einhaltung der ethischen Richtlinien}

Interessenkonflikt. Der korrespondierende Autor gibt für sich und seine Ko-Autoren an, dass kein Interessenkonflikt besteht.

\section{Literatur}

1. Earle CC (2012) It takes a village. J Clin Oncol 30(4):353-354

2. Muller M, Pfister D, Markett S et al (2009) [How many patient deaths can a team cope with? a nationwide survey of palliative care units in Germany]. Schmerz 23(6):600-608

3. Kleeberg U, Wedding U, Gärtner J et al (2011) Onkologie und Palliativmedizin: Eine Standortbestimmung. Info Onkologie 14(4):56-57

4. Cherny NI, Catane R, Kosmidis P (2003) ESMO takes a stand on supportive and palliative care. Ann Oncol 14(9):1335-1337

5. Smith TJ, Temin S, Alesi ER et al (2012) American Society of Clinical Oncology provisional clinical opinion: the integration of palliative care into standard oncology care. J Clin Oncol 30(8):880-887
Konrad K. Ruchter

K.K. Richter

Herausgeber

da. toforem

K. Höffken

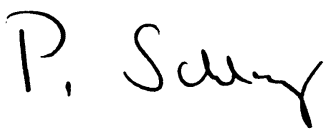

P.M. Schlag

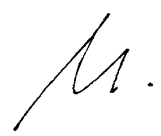

M. Bamberg

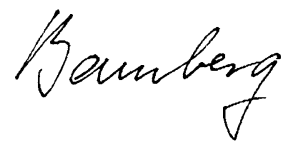

\section{Reinhold-Schwarz-Förderpreis für Psychoonkologie 2013 erst- mals verliehen}

Die Arbeitsgemeinschaft für Psychoonkologie in der Deutschen Krebsgesellschaft (PSO), der Verein für Fort- und Weiterbildung Psychosoziale Onkologie (WPO e. V.) sowie die Familie Schwarz haben am 22.11.2013 erstmals den ReinholdSchwarz-Förderpreis für Psychoonkologie vergeben. Preisträgerinnen 2013 sind die Diplompsychologinnen Hilke Rath und Sigrun Vehling, beide vom Universitätsklinikum Hamburg-Eppendorf, Institut und Poliklinik für Medizinische Psychologie, Hamburg.

Die Arbeit von Sigrun Vehling (Receiving palliative treatment moderates the effect of age and gender on demoralization in patients with cancer) untersucht in einer methodisch hochwertigen Studie die Einflussfaktoren für die Entstehung psychischer Folgeprobleme insbesondere der Demoralisierung bei Patienten in der palliativen Behandlung.

Die Arbeit von Hilke Rath (Work-related predictors of not returning to work after inpatient rehabilitation in cancer patients) untersucht den bisher wenig beforschten Bereich der beruflichen Wiedereingliederung von Krebspatienten. Hierzu wurden in komplexen Analysen Einflussfaktoren identifiziert, die die berufliche Wiedereingliederung erschweren.

Die Studien beider Preisträgerinnen sind in international hochrangigen Fachzeitschriften publiziert worden.

Die Preisverleihung erfolgte im Rahmen der 12. Jahrestagung der Arbeitsgemeinschaft für Psychoonkologie in der Deutschen Krebsgesellschaft e.V. (PSO) in Dresden. Der Preis ist mit insgesamt $3.000 €$ dotiert und wird jährlich ausgeschrieben.

Quelle: Arbeitsgemeinschaft für Psychoonkologie in der Deutschen Krebsgesellschaft e.V., www.pso-ag.de 